\title{
Spatial statistics of atmospheric particulate matter in China
}

\author{
Shenghui Gao ${ }^{\mathrm{a}}$, Yangjun Wang ${ }^{\mathrm{b}}$, Yongxiang Huang ${ }^{\mathrm{c}}$, Quan Zhou ${ }^{\mathrm{a}}$, \\ Zhiming $\mathrm{Lu}^{\mathrm{a}}$, Xiang Shi ${ }^{\mathrm{b}}$, Yulu Liu ${ }^{\mathrm{a}}$ \\ ${ }^{a}$ Shanghai Institute of Applied Mathematics and Mechanics, Shanghai Key Laboratory \\ of Mechanics in Energy Engineering, Shanghai University, Shanghai 200072, China \\ ${ }^{b}$ School of Environmental and Chemical Engineering, Shanghai University, Shanghai \\ 200444, China \\ ${ }^{c}$ State Key Laboratory of Marine Environmental Science, Xiamen University, Xiamen \\ 361102, China
}

\begin{abstract}
In this paper, the spatial dynamics of the atmospheric particulate matters (resp. $\mathrm{PM}_{10}$ and $\mathrm{PM}_{2.5}$ ) are studied using turbulence methodologies. It is found experimentally that the spatial correlation function $\rho(r)$ shows a log-law on the mesoscale range, i.e., $50 \leq r \leq 500 \mathrm{~km}$, with an experimental scaling exponent $\beta=0.45$. The spatial structure function shows a powerlaw behavior on the mesoscale range $90 \leq r \leq 500 \mathrm{~km}$. The experimental scaling exponent $\zeta(q)$ is convex, showing that the intermittent correction is relevant in characterizing the spatial dynamic of particulate matter. The measured singularity spectrum $f(\alpha)$ also shows its multifractal nature. Experimentally, the particulate matter is more intermittent than the passive scalar, which could be partially due to the mesoscale movements of the atmosphere, and also due to local sources, such as local industry activities. Keywords: Particulate matter, Logarithm spatial correlation, Multifractality
\end{abstract}




\section{Introduction}

In recent decades, many cities in China have experienced heavy air pollution episodes leading to negative impacts on human health Streets and Waldhoff, 2000; Chan and Yao, 2008; Matus et al., 2012; Chen et al., 2013; Wang et al., 2014b c; Zhang et al., 2014; Rohde and Muller, 2015), and the air pollution have been one of the biggest problems in urban areas of many megacities in China. The Jing-Jin-Ji region, Yangtze River Delta region, Pearl River Delta region, Central China region and Cheng-Yu region, to list a few, are the major polluted regions in China due to highly densed population and high energy consumption. In order to improve the air quality, China government issued new national ambient air quality standards in 2012 and were to be implemented in Jan.2016. According to the new standards, air quality indices ranging from 0 to 50 and ranging from 51 to 100 represent excellent and good, respectively. However, air quality index equal to or above 101 means the air quality does not meet the national ambient air quality standards. Hourly observed concentration data for pollutants in numerous cities were released by the government. According to the new ambient air quality standards, only 8 out of China's 74 biggest cities met the government's air quality standards in 2014 . Although not by much, the air quality in 2014 was better than in 2013: see http://www.mep.gov.cn/. Particulate matter with an aerodynamic diameter $10 \mu \mathrm{m}$ or less, or $\mathrm{PM}_{10}$ usually dominantes pollution episodes caused by dust storms. Particulate matter with a diameter less than $2.5 \mu \mathrm{m}$, or $\mathrm{PM}_{2.5}$ usually could lead to more serious health problems for local residents than coarse particle due to

\footnotetext{
*Corresponding author. Email: yjwang326@shu.edu.cn.

Email address: yongxianghuang@gmail.com (Yongxiang Huang)
} 
easier inhalation. Furthermore, most haze episodes occurring in China are characterized by high concentrations of $\mathrm{PM}_{2.5}$ in the ambient air.

Rohde and Muller (2015) applied the Kriging interpolation to four months of data to retrieve the pollution maps for eastern China and discovered that the greatest pollution occurs in the east. Air pollution episodes can cover a large region and are particularly intense in a northeast corridor that extends from outside of Shanghai to north of Beijing. Particulate matter is a very complicated mixture that comes from numerous emission sources. Industrial process was the dominant local contributor to PM2.5 concentration in the whole city of Shanghai except at the urban center where vehicle emissions contribute slightly more (Wang et al. 2014c). Moreover, haze episode could be caused by the combination of anthropogenic emissions, unusual atmospheric circulation, the depression of strong cold air activities, and weak boundary layer ventilation (Wang et al., 2014a). Wang et al. (2014b) concluded that the response of $\mathrm{PM}_{2.5}$ to meteorology possibly changes a feedback loop whereby planetary boundary layer dynamics amplify the initial perturbation of $\mathrm{PM}_{2.5}$.

Note that the air pollution occurred in the planetary boundary layer, where atmospheric turbulence is involved, showing a significant impact on the transport and dispersion of pollution matter. However, the effects of atmospheric turbulence are seldom studied in a multiscale view. A common behavior of the turbulence is the multiscaling, or multifractality, of the velocity field (Frisch, 1995). In the view of the hydrodynamic turbulence, a large range of spatial and temporal scales/freedoms are involved, resulting in a cascade process in which the energy transfers from large-scale structures to small-scale ones until the fluid viscosity converts the kinetic energy into 
heat. This phenomenological Kolmogorov-Richardson energy cascade picture has been widely and successfully applied in multiple disciplinary fields, such as financial activity (Schmitt et al., 1999, Ghashghaie et al., 1996, Li and Huang, 2014), crack of rock surfaces (Schmittbuhl et al., 1995), rainfall patterns (Tessier et al., 1996), etc.

Specifically for atmospheric turbulence, due to the geometrical constrain of the atmospheric movement, there exists several typical spatial scales. These include the microscale (resp. $1 \mathrm{~km}$ or less), showing threedimensional property and synoptic scale (resp. up to $1000 \mathrm{~km}$ ), showing a two-dimensional feature (Vallgren et al., 2011). Between the microscale and synoptic scale, a large range of scale motion exists in mesoscale structures (resp. from few dozens of $\mathrm{km}$ to few hundreds $\mathrm{km}$ ). The famous Kolmogorov 5/3-law has been observed on the mesoscale range Nastrom et al., 1984; Nastrom and Gage, 1985) and agrees well with the above mentioned Kolmogorov-Richardson cascade prediction (Vallgren et al., 2011). Therefore, the air pollution indices, such as $\mathrm{PM}_{2.5}$ could display a spatial scaling behavior since they are advected mainly by these mesoscale structures. In this paper, we employ the standard structure function analysis to retrieve the multiscale and multiscaling properties of the $\mathrm{PM}_{10}$ and $\mathrm{PM}_{2.5}$ to show the impact from the mesoscale atmospheric turbulence.

\section{Data}

The hourly concentrations of PM2.5 and PM10 were released by the government (http://www.cnemc.cn). We processed these data into daily average concentrations to be used in this study. There are 305 monitor stations belonging to different cities. Fig 1 a) shows the spatial distribution 

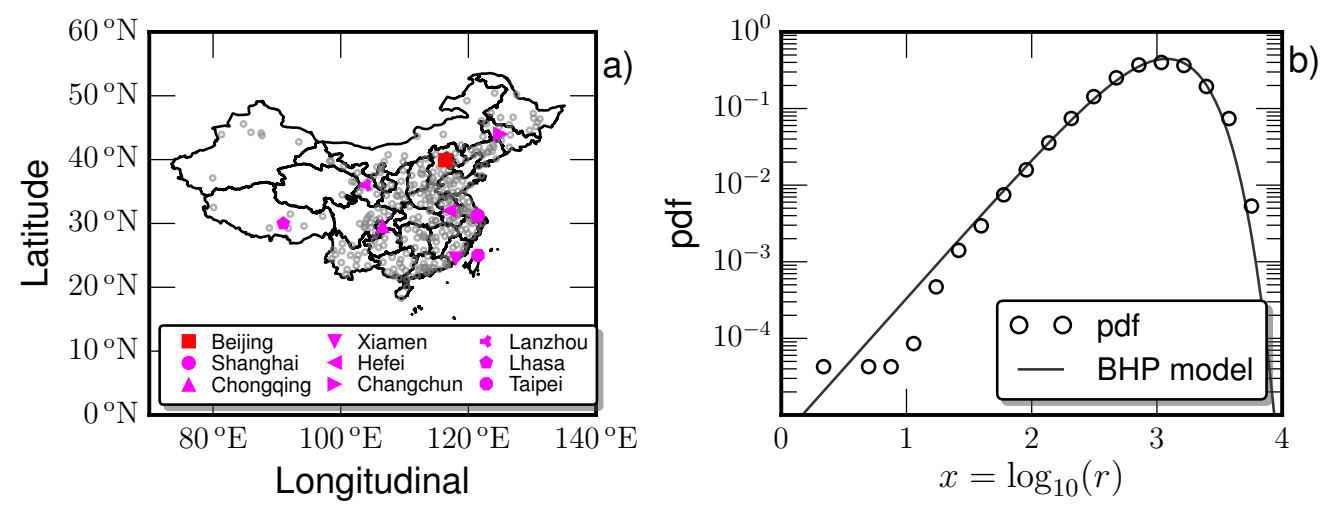

Figure 1: a) The spatial distribution of 305 monitor stations in different cities. b) The distribution of the neighbour distances $(\bigcirc)$. The BHP model with parameters $b=0.938$, and $K=2.14$ obtained numerically is illustrated by a solid line.

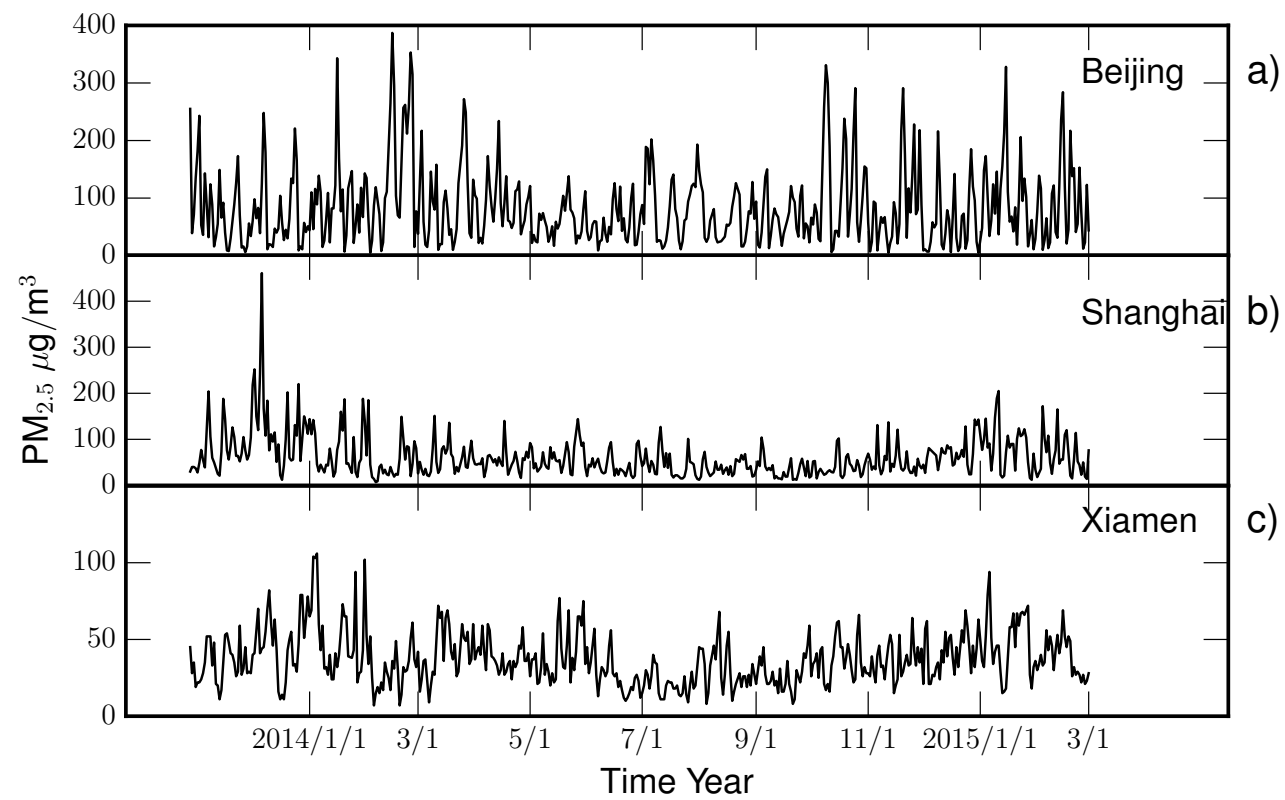

Figure 2: Illustration of the recorded $\mathrm{PM}_{2.5}$ in $\mu \mathrm{g} / \mathrm{m}^{3}$ of three typical cities: a) Beijing, b) Shanghai and c) Xiamen. Large variance is visible, showing the intermittency pattern.

of these monitor stations, which were monitored during the period from 31 Dec. 2013 to 01 Mar. 2015, corresponding to 425 days for most of cities with 
several missing. In total, there are 82,755 daily averaged data points. The neighbor distance, $r$, of two cities is calculated via a great circle distance algorithm. The corresponding probability density function (pdf) is shown in Fig, 1 b). For convenience, we used the logarithm of $r, x=\log _{10}(r)$. A bin width 0.1 in the logarithm scale was adopted to estimate the pdf. It is interesting to note that the measured pdf agrees well with the BramwellHoldsworth-Pinton (BPH) formula (Bramwell et al., 1998), which is:

$$
\Pi(y)=K\left(e^{y-e^{y}}\right)^{a}, y=b(x-s), a=\pi / 2,
$$

where parameters $b=0.938$, and $K=2.14$ were obtained numerically (Bramwell et al., 2000). Note that this formula was first introduced to characterize rare fluctuations in turbulence and critical phenomena. The neighbor distance was often chosen based on cities located near a water source. Therefore, this neighbor distance could be used as a proxy of the spatial distribution of water sources. However, this postulate needs to be verified by carefully analyzing neighbor distance statistics for different regions. Fig. 2 shows the recorded $\mathrm{PM}_{2.5}$ index with unit $\mu \mathrm{g} / \mathrm{m}^{3}$ for three typical cities, Beijing, Shanghai and Xiamen. Visually, the measured index shows similar evolution trends: they are higher during the winter and smaller during the summer, showing an annual cycle. In the following analysis, these database are analyzed by pairing two cities, i.e., $\left[\theta_{i}(t), \theta_{j}(t)\right]$ with the neighbor distance $r_{i j}$, where $\theta_{i}(t)$ is the air quality index of the $i$ th city. 


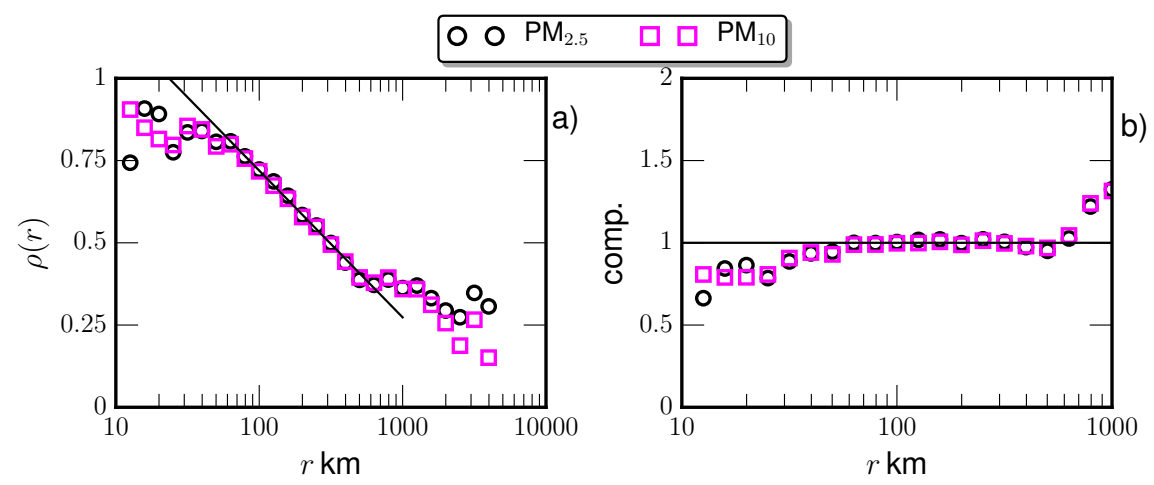

Figure 3: (Color online) a) Measured spatial correlation function $\rho(r)$ of $\mathrm{PM}_{2.5}(\bigcirc)$ and $\mathrm{PM}_{10}(\square)$. A log-law is observed on the range $50 \leq r \leq 500 \mathrm{~km}$ with an scaling exponent $\beta=0.45 \pm 0.02$. b) The corresponding compensated curve using the fitted parameters to emphasize the experimental log-law behavior.

\section{Results}

\subsection{Spatial correlation}

We first calculated the spatial correlation function for different neighbor distances $r$. The spatial correlation $\rho(r)$ is defined by the following equation:

$$
\rho(r)=\frac{1}{N(r)} \sum^{N(r)} \frac{\left\langle\tilde{\theta}_{i}(t) \tilde{\theta}_{j}(t) \mid r_{i j}=r\right\rangle_{t}}{\sigma_{i} \sigma_{j}},
$$

where $\tilde{\theta}_{i}(t)=\theta_{i}(t)-\left\langle\theta_{i}(t)\right\rangle_{t}$ is the centered index of the $i$ th city, \langle\rangle$_{t}$ is the time average, $\sigma_{i}$ is the standard deviation, $r$ is the neighbor distance; and $N(r)$ is the number of pairs with distance $r$, where a bin width 0.1 in the logarithm scale is used. The final $\rho(r)$ is then calculated for all pairs of cities with distance $r$. Fig. 3a) shows the measured $\rho(r)$ in a semilog plot for the $\mathrm{PM}_{2.5}(\bigcirc)$ and $\mathrm{PM}_{10}(\square)$. A log-law is observed in the range $50 \leq r \leq 500 \mathrm{~km}$, as follows:

$$
\rho(r) \propto A-\beta \log _{10}(r)
$$


where $\beta$ is the scaling exponent, which is experimentally $\beta=0.45 \pm 0.02$. To emphasize the experimental log-law behavior, Fig. $3 \mathrm{~b}$ ) shows the corresponding compensated curve using the fitted parameters. A clear plateau confirms the existence of the log-law. Note that the log-law range is between the microscale (resp. $1 \mathrm{~km}$ or less) and synoptic scale (resp. up to $1000 \mathrm{~km}$ ), corresponding to the mesoscale movement in the atmospheric boundary layer (Vallgren et al., 2011).

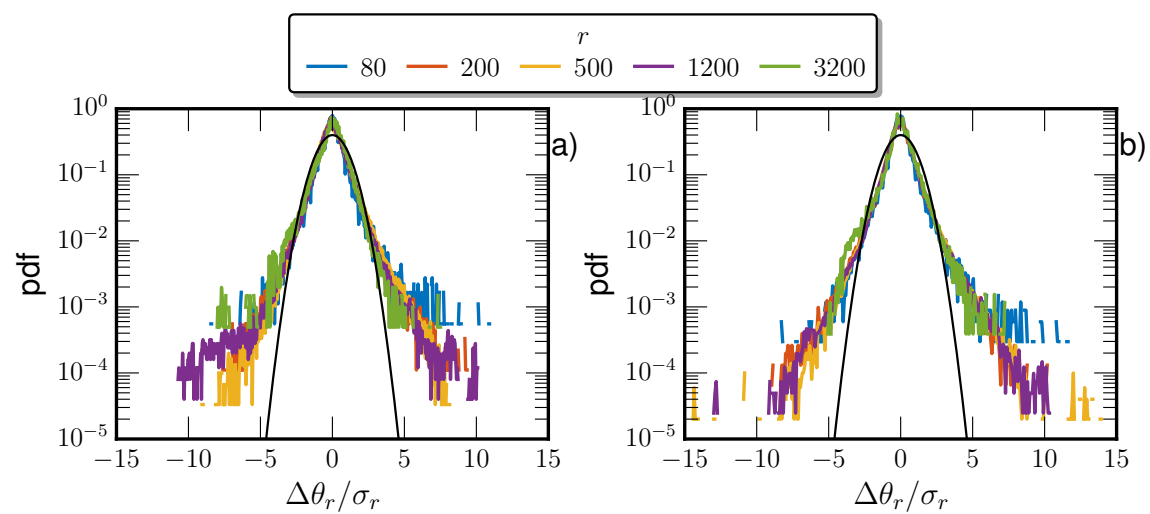

Figure 4: Experimental probability density function of the increment $\Delta \theta_{r} / \sigma_{r}$ for various separation scales $r$ : a) $\mathrm{PM}_{10}$ and b) $\mathrm{PM}_{2.5}$. For comparison, the normal distribution is illustrated by a thin solid line. The core part, i.e., $-2.5 \leq \Delta \theta_{r} / \sigma_{r} \leq 2.5$ can be fitted by an exponential law with a slope $0.67 \pm 0.02$. The tail, e.g., $\left|\Delta \theta_{r} / \sigma_{r}\right| \geq 4$ has an exponential trend with fitted slope $0.35 \pm 0.03$.

\subsection{Structure Function Analysis}

Intermittency or multifractality is an important feature of the turbulentlike dynamical systems (Frisch, 1995). More precisely, numerous spatial or temporal freedoms exist simultaneously and interact with each other to transfer energy, momentum, or other physical quantities. To characterize this multiscale interaction, structure function analysis is used to retrieve the 


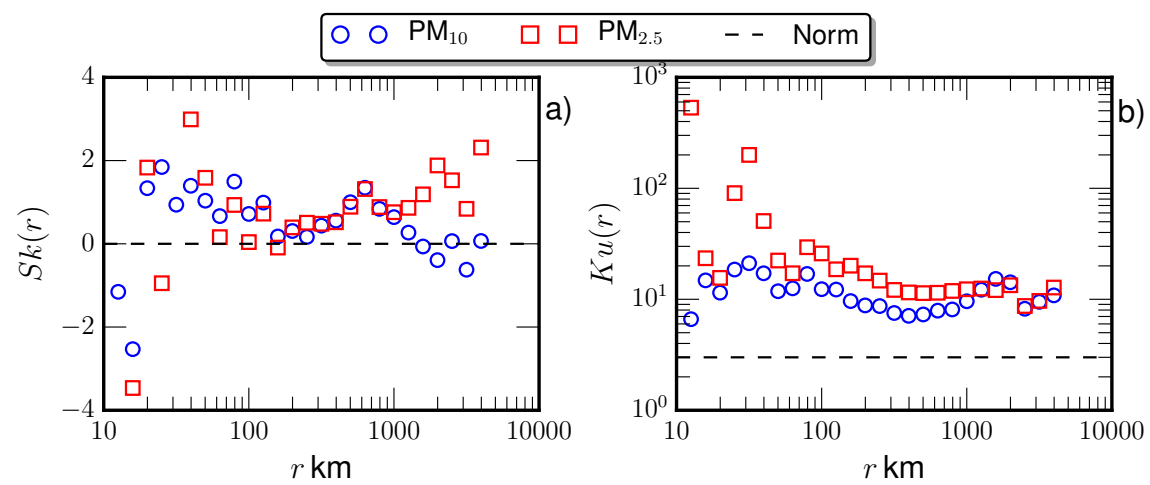

Figure 5: Measured a) skewness $S k(r)$ and b) kurtosis $K u(r)$ for $\mathrm{PM}_{10}(\bigcirc)$ and $\mathrm{PM}_{2.5}$ $(\square)$. For comparison, the corresponding value of the normal distribution is illustrated by a dashed line.

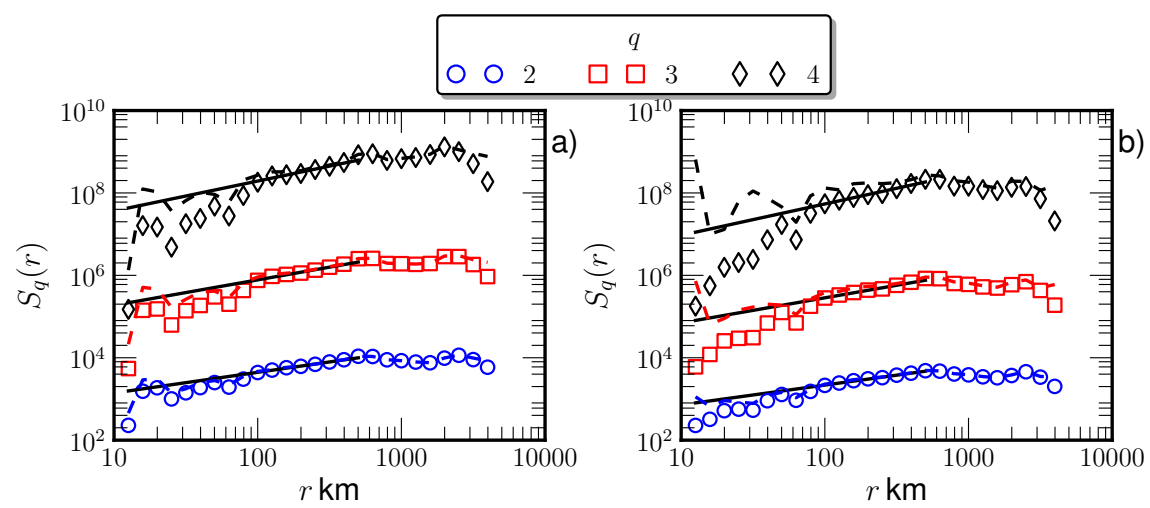

Figure 6: (Color online) Measured high-order structure function $S_{q}(r)$ : a) $\mathrm{PM}_{10}$ and b) $\mathrm{PM}_{2.5}$. The dashed lines are the raw structure functions. The symbols are the functions without contamination of rare events. Power-law behavior is observed in the mesoscale range $90 \leq r \leq 500 \mathrm{~km}$. The solid line is a power-law fitting.

scale invariance for high Reynolds turbulent flows (Kolmogorov, 1941). It is then widely used in a variety of fields, including financial activity (Schmitt) et al., 1999; Ghashghaie et al., 1996; Li and Huang, 2014), crack of rock surfaces (Schmittbuhl et al., 1995), rainfall patterns (Tessier et al., 1996), etc., to retrieve the scale invariant parameters. 


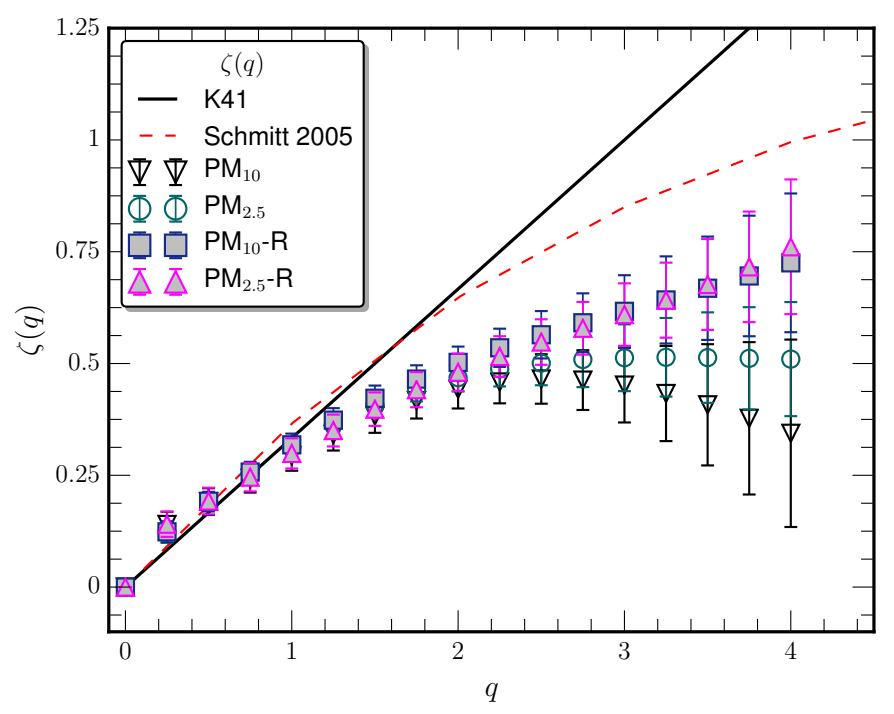

Figure 7: Measured scaling exponents $\zeta(q)$ for $\mathrm{PM}_{2.5}$ and $\mathrm{PM}_{10}$ with and without (denoted as $\mathrm{PM}_{2.5}-\mathrm{R}$ or $\mathrm{PM}_{10}-\mathrm{R}$ ) rare events. For comparison, the Kolmogorov value $q / 3$ and the complied scaling exponents for the passive scalar (dashed line) are also shown.

To characterize the interaction between different scales, the $q$ th-order structure function is defined as follows:

$$
S_{q}(r)=\left\langle\left|\Delta \theta_{r}\right|^{q}\right\rangle_{t},
$$

where $\Delta \theta_{r}=\left\{\theta_{i}(t)-\theta_{j}(t)\right\} \mid r_{i j=r}$ is a set of the increment; and $r$ is the separation scale (resp. neighbor distance in this work). Note that the increment set $\Delta \theta_{r}$ is first calculated for all pairs with distance $r$. Then, the $q$ th-order structure function $S_{q}(r)$ is estimated. In the case of scale invariance, the structure function exhibits a power-law behavior:

$$
S_{q}(r) \sim r^{\zeta(q)}
$$

where $\zeta(q)$ is the scaling exponent. For the turbulent velocity and passive scalar, the corresponding Kolmogorov scaling exponent without in- 


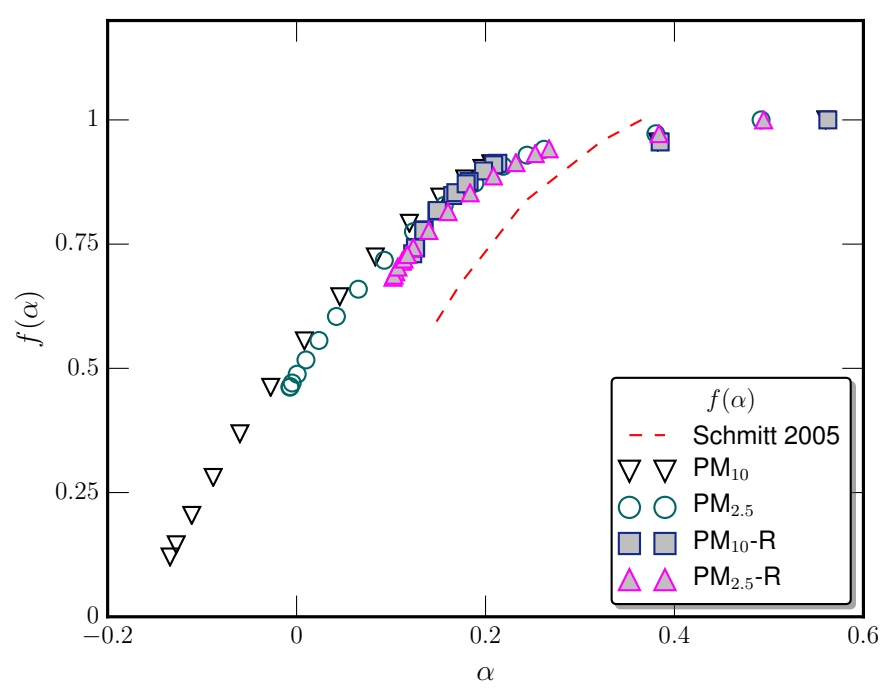

Figure 8: Experimental singularity spectrum $f(\alpha)$ with and without the rare events. For comparison, the singularity spectrum for the passive scalar on the range $0 \leq q \leq 4$ is also presented by dashed line.

termittent correction is $\zeta(q)=q / 3($ Frisch, 1995). However, both experiments and numerical simulations show that the measured $\zeta(q)$ deviates from $q / 3$ Anselmet et al., 1984; Sreenivasan and Antonia, 1997; Frisch, 1995: Warhaft, 2000; Huang et al., 2010). It is then recognized as the intermittent nature of the dissipation field (Kolmogorov, 1962; Frisch, 1995). A concept of multifractality is then put forward to interpret this break in the self-similarity (Parisi and Frisch, 1985; Benzi et al., 1984). It was determined that the multifractality is one of the most important features of the turbulent-like dynamic systems.

Fig. 4 shows the measured pdf of the increment $\Delta \theta_{r} / \sigma_{r}$ on several separation scales, where $\sigma_{r}$ is a standard deviation of the $\Delta \theta_{r}$. For comparison, the normal distribution is illustrated by a thin solid line. Roughly speaking, all these pdfs collapse with each other, not only for different separation 
scales, but also for $\mathrm{PM}_{10}$ and $\mathrm{PM}_{2.5}$ (not shown here). They can be further divided into three parts: the core part $\left(\left|\Delta \theta_{r} / \sigma_{r}\right| \leq 2.5\right)$, the tail part with rare events $\left(\left|\Delta \theta_{r} / \sigma_{r}\right| \geq 4\right)$, and the transition range, $2.5 \leq\left|\Delta \theta_{r} / \sigma_{r}\right| \leq 4$. The first two parts can be fitted using an exponential distribution with slopes $0.67 \pm 0.02$ and $0.35 \pm 0.03$, respectively. To compare to the normal distribution, we calculated the skewness and kurtosis as follows:

$$
S k(r)=\frac{\left\langle\Delta \theta_{r}^{3}\right\rangle}{\sigma_{r}^{3}}, K u(r)=\frac{\left\langle\Delta \theta_{r}^{4}\right\rangle}{\sigma_{r}^{4}}
$$

The skewness, $S k(r)$, indicates an asymmetric shape of experimental pdf, where $S K=0$ if the pdf is symmetric. The kurtosis $K u(r)$ is used to characterize the deviation from the normal distribution. Fig. 5 shows the measured a) $S k(r)$ and b) $K u(r)$, where the value of the normal distribution is illustrated by a dashed line. Visually, these two statistics exhibits a scaledependence, indicating potential intermittency.

Fig. 6 shows the experimental high-order structure functions, $S_{q}(r)$, up to $q=4$ for a) $\mathrm{PM}_{10}$ and b) $\mathrm{PM}_{2.5}$. To avoid a possible contamination of rare events, the structure functions were also calculated after removing the values where the histogram contains less then 10 data points; the corresponding $S_{q}(r)$ is shown as symbols. Power-law behavior is observed in the range $90 \leq$ $r \leq 500 \mathrm{~km}$. The scaling exponent $\zeta(q)$ was then calculated on this range using a least square fitting algorithm. Fig. 7] shows the measured scaling exponent, $\zeta(q)$, in which the errorbar indicates a $95 \%$ fitting confidence. For comparison, the Kolmogorov non-intermittent value $q / 3$ (solid line) and the value for passive scalar compiled by Schmitt (2005) (dashed line) are also shown. When $q \leq 2$, the measured $\zeta(q)$ are almost the same for $\mathrm{PM}_{10}$ and $\mathrm{PM}_{2.5}$ with or without rare events. When $q>2$, the value with rare 
events bends down and deviates more from $q / 3$, showing a more intermittent statistics. We also note that due to the rare events the scaling exponents of $\mathrm{PM}_{10}$ are more intermittent than those of $\mathrm{PM}_{2.5}$.

\section{Discussion}

To characterize the intensity of the intermittency or multifractality, we calculate the singularity spectrum $f(\alpha)$ via the Legendre transform, i.e.,

$$
\alpha=\frac{d \zeta(q)}{d q}, f(\alpha)=\min _{q}\{q \alpha-\zeta(q)+1\}
$$

where $\alpha$ is the generalized Hurst number or intensity of multifractality. The broader measured $\alpha$ and $f(\alpha)$ are, the more intermittent the field is (Frisch, 1995). This singularity spectrum was first introduced in the 1980s to characterize the multifractality of the turbulence and chaotic systems (Parisi and Frisch, 1985, Benzi et al., 1984). Fig. 8 shows the measured $f(\alpha)$ versus $\alpha$. For comparison, the singularity spectrum was calculated from the compiled passive scalar scaling exponent in the range $0 \leq q \leq 4$ and is shown as a dashed line. Visually, the measured $\alpha$ and $f(\alpha)$ conform to the existence of the multifractality. Furthermore, by removing the rare events, the lower part of the singularity spectrum is suppressed since it corresponds to the scaling exponents $\zeta(q)$ shown in Fig.7. However, the singularity spectrum either with or without the rare events (resp. strong local events) is more intermittent than the passive scalar (Warhaft, 2000).

To simplify the discussion, we considered here the particulate matter as a continuous media, whereas $\theta(x, t)$ is a function of spatial coordinate, $x$, and time, $t$, and an analogy to the passive scalar turbulence. The governing 
equation for the atmospheric particulate matter is then written as:

$$
\frac{\partial \theta(x, t)}{\partial t}+\mathbf{u}(x, t) \cdot \nabla \theta(x, t)=\kappa \nabla^{2} \theta(x, t)+\mathbf{f}_{\theta}(x, t)
$$

where $\kappa$ is the mass diffusion coefficient; $\mathbf{f}_{\theta}(x, t)$ is the external forcing term (resp. the local sources); $\nabla^{2}$ is the Laplace operator. This equation is a mass transport equation indicating that $\theta(x, t)$ is mainly advected by $\mathbf{u}(x, t)$. Note that $\mathbf{f}_{\theta}(x, t)$ is different for different regions and time. For example, during winter, one main source of the atmospheric particulate matter is the coal consumption for heating in the northern China. Due to the advection of the atmospheric mesoscale structures, the second-term in the l.h.s thus induces scaling behavior. The measured scaling exponent $\zeta(q)$ shows more intermittent nature than the exponent for passive scalar turbulence. This variance could be an effect of the local emission towards air pollution, or it could be other mechanisms that are ignored in the governing Equation (8).

\section{Conclusion}

In summary, we present an analysis of the spatial statistics of particulate matter in China in this paper. We determined that the neighbor distance, $r$, can be described by the BHP formula without tuning any parameters. The experimental spatial correlation function $\rho(r)$ obeys a log-law behavior in the mesoscale range $50 \leq r \leq 500 \mathrm{~km}$ with an experimental scaling exponent 0.45. Additionally, the high-order structure functions obey powerlaw behavior in the range $90 \leq r \leq 500 \mathrm{~km}$. The retrieved scaling exponent $\zeta(q)$ curves were determined to be convex, showing the multifractal nature of the particulate matter. Moreover, the findings from these exponents demonstrate a more intermittent dynamics than the exponents for passive 
scalar turbulence. The results presented in this paper provide a better understanding of the multiscale dynamics of air pollution, especially for the particulate matter advected by the mesoscale structures in the atmosphere.

\section{Acknowledgements}

This work is partially sponsored by the National Natural Science Foundation of China (NSFC) under Grant No. 11202122, 11222222, 11301433 and 11332006, and the Fundamental Research Funds for the Central Universities (Grant No. 20720150075). This work is partially financially supported by the Jinhua EPB's Funds (Grant No: YG2014-FW673-ZFCG046). The analyzed data and a MATLAB source code package are available at: https://github.com/lanlankai.

\section{References}

\section{References}

Anselmet, F., Gagne, Y., Hopfinger, E. J., Antonia, R. A., 1984. High-order velocity structure functions in turbulent shear flows. J. Fluid Mech. 140, 63-89.

Benzi, R., Paladin, G., Vulpiani, A., Parisi, G., 1984. On the multifractal nature of fully developed turbulence and chaotic systems. J. Phys. A 17, 3521-3531.

Bramwell, S., Christensen, K., Fortin, J.-Y., Holdsworth, P., Jensen, H., Lise, S., López, J., Nicodemi, M., Pinton, J.-F., Sellitto, M., 2000. Universal fluctuations in correlated systems. Phys. Rev. Lett. 84 (17), 3744.

Bramwell, S., Holdsworth, P., Pinton, J.-F., 1998. Universality of rare fluctuations in turbulence and critical phenomena. Nature 396 (6711), 552-554.

Chan, C. K., Yao, X., 2008. Air pollution in mega cities in china. Atmos. Environ. 42 (1), $1-42$.

Chen, R., Zhao, Z., Kan, H., 2013. Heavy smog and hospital visits in beijing, china. Am. J. Respir. Crit. Care Med. 188 (9), 1170-1171. 
Frisch, U., 1995. Turbulence: the legacy of AN Kolmogorov. Cambridge University Press.

Ghashghaie, S., Breymann, W., Peinke, J., Talkner, P., Dodge, Y., 1996. Turbulent cascades in foreign exchange markets. Nature 381 (6585), 767-770.

Huang, Y., Schmitt, F., Lu, Z., Fougairolles, P., Gagne, Y., Liu, Y., 2010. Second-order structure function in fully developed turbulence. Phys. Rev. E 82 (2), 026319.

Kolmogorov, A., 1962. A refinement of previous hypotheses concerning the local structure of turbulence in a viscous incompressible fluid at high Reynolds number. J. Fluid Mech. $13,82-85$.

Kolmogorov, A. N., 1941. Local structure of turbulence in an incompressible fluid at very high Reynolds numbers. Doklady Akademii Nauk 30, 301.

Li, M., Huang, Y., 2014. Hilbert-Huang Transform based multifractal analysis of China stock market. Physica A 406, 222-229.

Matus, K., Nam, K.-M., Selin, N. E., Lamsal, L. N., Reilly, J. M., Paltsev, S., 2012. Health damages from air pollution in china. Global Environ. Chang. 22 (1), 55-66.

Nastrom, G., Gage, K., Jasperson, W., 1984. Kinetic energy spectrum of large-and mesoscale atmospheric processes. Nature 310 (5972), 36-38.

Nastrom, G., Gage, K. S., 1985. A climatology of atmospheric wavenumber spectra of wind and temperature observed by commercial aircraft. J. Atmos. Sci. 42 (9), 950-960.

Parisi, G., Frisch, U., 1985. On the singularity spectrum of fully developed turbulence. Turbulence and predictability in geophysical fluid dynamics, North Holland, Proceedings of the International Summer School in Physics Enrico Fermi, 84-87.

Rohde, R. A., Muller, R. A., 2015. Air pollution in china: Mapping of concentrations and sources. PLoS One 10 (8), e0135749.

Schmitt, F., 2005. Relating Lagrangian passive scalar scaling exponents to Eulerian scaling exponents in turbulence. EPJ B 48 (1), 129-137.

Schmitt, F. G., Schertzer, D., Lovejoy, S., 1999. Multifractal analysis of foreign exchange data. Appl. Stochastic Models Data Anal. 15 (1), 29-53.

Schmittbuhl, J., Schmitt, F. G., Scholz, C., 1995. Scaling invariance of crack surfaces. J. Geophys. Res. 100 (B4), 5953-5973.

Sreenivasan, K., Antonia, R., 1997. The phenomenology of small-scale turbulence. Annu. Rev. Fluid Mech. 29, 435-472. 
Streets, D., Waldhoff, S., 2000. Present and future emissions of air pollutants in china::

So2, nox, and co. Atmos. Environ. 34 (3), 363-374.

Tessier, Y., Lovejoy, S., Hubert, P., Schertzer, D., Pecknold, S., 1996. Multifractal analysis and modeling of rainfall and river flows and scaling, causal transfer functions. j. Geophys. Res. 101, 26427-26440.

Vallgren, A., Deusebio, E., Lindborg, E., Dec 2011. Possible explanation of the atmospheric kinetic and potential energy spectra. Phys. Rev. Lett. 107, 268501.

Wang, H., An, J., Shen, L., Zhu, B., Pan, C., Liu, Z., Liu, X., Duan, Q., Liu, X., Wang, Y., 2014a. Mechanism for the formation and microphysical characteristics of submicron aerosol during heavy haze pollution episode in the yangtze river delta, china. Sci. Total Environ. 490, 501-508.

Wang, J., Wang, S., Jiang, J., Ding, A., Zheng, M., Zhao, B., Wong, D. C., Zhou, W., Zheng, G., Wang, L., Pleim, J., Hao, J., 2014b. Impact of aerosol-meteorology interactions on fine particle pollution during china severe haze episode in january 2013. Environ. Res. Lett. 9 (9), 094002.

Wang, Y., Li, L., Chen, C., Huang, C., Huang, H., Feng, J., Wang, S., Wang, H., Zhang, G., Zhou, M., Cheng, P., Wu, M., Sheng, G., Fu, J., Hu, Y., Russell, A. G., Wumaer, A., 2014c. Source apportionment of fine particulate matter during autumn haze episodes in shanghai, china. J. Geophys. Res. 119 (4), 1903-1914.

Warhaft, Z., 2000. Passive scalars in turbulent flows. Annu. Rev. Fluid Mech. 32 (1), 203-240.

Zhang, L.-W., Chen, X., Xue, X.-D., Sun, M., Han, B., Li, C.-P., Ma, J., Yu, H., Sun, Z.-R., Zhao, L.-J., Zhao, B.-X., Liu, Y.-M., Chen, J., Wang, P. P., Bai, Z.-P., Tang, N.J., 2014. Long-term exposure to high particulate matter pollution and cardiovascular mortality: a 12-year cohort study in four cities in northern china. Environ. Int. 62, $41-47$. 